\title{
A CLASS OF ARCWISE CONNECTED CONTINUA
}

\author{
CHARLES L. HAGOPIAN
}

Abstract. It is known that every bounded semi-aposyndetic plane continuum which does not separate the plane is arcwise connected. To show that this theorem remains true if the phrase "does not separate the plane" is replaced by "does not have infinitely many complementary domains" is the primary purpose of this paper.

Let $M$ be a continuum (a closed connected point set) and let $x$ and $y$ be distinct points of $M$. If $M$ contains a continuum $H$ and an open set $G$ such that $x \in G \subset H \subset M-\{y\}$, then $M$ is said to be aposyndetic at $x$ with respect to $y$. If $M$ is aposyndetic at $x$ with respect to each point of $M-\{x\}$, then $M$ is said to be aposyndetic at $x$. F. Burton Jones has shown that if $M$ is a bounded plane continuum which is aposyndetic (that is, aposyndetic at each of its points) and does not have infinitely many complementary domains, then $M$ is locally connected and therefore arcwise connected [4, Theorem 10]. The notion of aposyndesis can be generalized as follows.

Definition. A continuum $M$ is said to be semi-aposyndetic if for each pair of distinct points $x$ and $y$ of $M, M$ is aposyndetic either at $x$ with respect to $y$ or at $y$ with respect to $x$.

In a recent paper [3], the author established the arcwise connectedness property for bounded semi-aposyndetic nonseparating plane continua. For a sketch of the proof of this theorem see [2]. There are nonlocally connected continua, hence continua which do not have the conditions specified in Jones's theorem, which have these properties. However, the arcwise connectedness implication of Jones's theorem is not generalized by this result. The purpose of this paper is to extend the author's result to a class of continua which includes those studied by Jones. Here it is proved that if $M$ is a bounded semiaposyndetic plane continuum which does not have infinitely many complementary domains, then $M$ is arcwise connected.

Throughout this paper $S$ is the set of points of a simple closed surface (that is, a 2-sphere).

\footnotetext{
Presented to the Society, November 21, 1970; received by the editors October 16 1970.

AMS 1969 subject classifications. Primary 5475, 5455; Secondary 5422.

Key words and phrases. Arcwise connected continua, aposyndesis, semi-aposyndesis, cut point, complementary domain, and Jones's cyclic property.
} 
Theorem 1. Suppose $M$ is a continuum in $S$ and $S-M$ does not have infinitely many components. Let $D$ be the interior of a disk in $S$. Suppose $F$ is a component of $M-D$ and $x$ and $y$ are points of $F$ such that $F$ is not aposyndetic at $x$ with respect to $y$. Then $M$ is not aposyndetic at $x$ with respect to $y$.

Proof. Assume $M$ is aposyndetic at $x$ with respect to $y$. There exists a continuum $H$ and a region $V$ containing $y$ such that $x$ is in the interior of $H$ relative to $M$ and $H$ is in $M-\mathrm{Cl} V(\mathrm{Cl} V$ is the closure of $V$ ). Let $U_{1}$ and $V_{1}$ be circular regions in $S$ having radii less than 1 centered on $x$ and $y$ respectively such that $U_{1} \cap M \subset H, V_{1}$ $C V$, and $\mathrm{Cl} U_{1} \cap \mathrm{Cl} V_{1}=\varnothing$. The component of $F-V_{1}$ which contains $x$ is not open (relative to $F$ ) at $x$. Hence $\operatorname{Bd} U_{1}$ (the boundary of $U_{1}$ ) contains an arc-segment $I_{1}$ whose endpoints $a_{1}$ and $b_{1}$ lie in different components of $F-V_{1}$ such that $F \cap I_{1}=\varnothing$. There exists a simple closed curve $J_{1}$ which separates $a_{1}$ from $b_{1}$ and contains no point of $M-\left(D \cup V_{1}\right)$. In $J_{1} \cup I_{1}$ there exists a simple closed curve $K_{1}$ which separates $a_{1}$ from $b_{1}$ and contains no point of $F-V_{1}$ such that $K_{1} \cap I_{1}$ is connected. There is an arc-segment $T_{1}$ in $K_{1}$ which crosses $I_{1}$, lies in $S-V_{1}$, and has its endpoints $c_{1}$ and $d_{1}$ in Bd $V_{1} \cap((S-M) \cup D)$. Let $U_{2}$ and $V_{2}$ be circular regions having radii less than $\frac{1}{2}$ centered on $x$ and $y$ respectively such that $\mathrm{Cl} U_{2} \subset U_{1}-T_{1}$ and $\mathrm{Cl} V_{2} \subset V_{1}$. The component of $F-V_{2}$ which contains $x$ is not open (relative to $F$ ) at $x$. Hence there exists an arc-segment $I_{2}$ in Bd $U_{2}$ whose endpoints $a_{2}$ and $b_{2}$ lie in different components of $F$ $-V_{2}$ such that $F \cap I_{2}=\varnothing$. There exists a simple closed curve $K_{2}$ which separates $a_{2}$ from $b_{2}$ and contains no point of $F-V_{2}$ such that $K_{2} \cap I_{2}$ is connected and $\left(K_{2}-I_{2}\right) \cap\left(M-\left(V_{2} \cup D\right)\right)=\varnothing$. In $K_{2}-V_{2}$ there is an arc-segment $T_{2}$ which crosses $I_{2}$ and has its endpoints $c_{2}$ and $d_{2}$ in $\mathrm{Bd} V_{2} \cap((S-M) \cup D)$. Continue this process. For each $n=3,4,5, \cdots$, there exist circular regions $U_{n}, V_{n}$, arc-segments $I_{n}, T_{n}$, and a simple closed curve $K_{n}$ such that (1) $U_{n}$ and $V_{n}$ are centered on $x$ and $y$ respectively and each have radius less than $1 / n$, (2) $\mathrm{Cl} U_{n} \subset U_{n-1}-\bigcup_{i=1}^{n-1} T_{i}$ and $\mathrm{Cl} V_{n} \subset V_{n-1}$, (3) $I_{n}$ is in $\mathrm{Bd} U_{n}-F$ and has endpoints $a_{n}$ and $b_{n}$ in $F$, (4) $K_{n}$ separates $a_{n}$ from $b_{n}$ in $S$, (5) $K_{n}$ $C(S-F) \cup V_{n}$ and $K_{n} \cap I_{n}$ is connected, (6) $T_{n} \subset K_{n}-V_{n}$ and $T_{n}$ $\cap I_{n} \neq \varnothing$, and (7) the endpoints $c_{n}$ and $d_{n}$ of $T_{n}$ are in Bd $V_{n} \cap((S-M) \cup D)$.

Since $M$ has only a finite number of complementary domains, it may be assumed without loss of generality that there exist complementary domains $C$ and $G$ of $M$ such that $\left\{c_{1}, c_{2}, c_{3}, \ldots\right\}$ is in $C \cup D$ and $\left\{d_{1}, d_{2}, d_{3}, \cdots\right\}$ is in $G \cup D$ (here it may be necessary to delete 
certain elements of the original sequences and rename others). Suppose $\left\{c_{1}, c_{2}, c_{3}, \cdots\right\} \cap D$ is infinite. Assume without loss of generality that $\left\{c_{1}, c_{2}, c_{3}, \cdots\right\}$ is contained in $D$. Since $c_{1}, c_{2}, c_{3}, \cdots$ converges to $y, y$ belongs to the boundary of the disk $\mathrm{Cl} D$. There exists a region $E$ in $V_{1}$ containing $y$ such that $E \cap D$ is the interior of a disk in $S[6$, Theorem 6, p. 163]. Note that $E \cap D$ contains all but finitely many points of $c_{1}, c_{2}, c_{3}, \cdots$. Suppose, without loss of generality, that $\left\{c_{1}, c_{2}, c_{3}, \cdots\right\}$ is a subset of $E \cap D$. The set $D$ $\cap\left\{d_{1}, d_{2}, d_{3}, \ldots\right\}$ is finite; for otherwise, there would exist an integer $j$ such that $\left\{c_{j}, d_{j}\right\} \subset E \cap D$ and $T_{j} \cup(E \cap D)$ would separate $a_{j}$ from $b_{j}$ which contradicts the assumption that $F$ is a component of $M-D$ [6, Theorem 32, p. 181]. Furthermore, if $i$ and $j$ are positive integers $(i<j)$, then $F \cup I_{j}$ separates $d_{i}$ from $d_{j}$ in $S$; for if this were not the case, $S-\left(F \cup I_{j}\right)$ would contain an arc $A$ which goes from $d_{i}$ to $d_{j}$ and $(E \cap D) \cup A \cup T_{i} \cup T_{j}$ would separate $a_{j}$ from $b_{j}$, again contradicting the assumption that $F$ is a component of $M-D$. Hence there exist integers $i$ and $j(i<j)$ such that $\left\{d_{i}, d_{j}\right\} \subset S-M$ and $F \cup I_{j}$ separates $d_{i}$ from $d_{j}$ in $S$. Since $d_{i}$ and $d_{j}$ are in the same complementary domain of $M$, there exists an arc $B$ in $S-M$ which goes from $d_{i}$ to $d_{j}$. Let $Z$ denote the complementary domain of $F \cup I_{j}$ which contains $d_{j}$. Let $k$ denote the first point of $B \cap B d V_{i} \cap Z$ and let $h$ be the last point of $B \cap \mathrm{Bd} V_{i}$ which precedes $k$ with respect to the order on $B$. Let $L$ be the subarc of $B$ which has endpoints $h$ and $k$. Note that $h$ does not belong to $Z$ and $L \cap C l V_{i}=\{h, k\} .\left(F \cup I_{j}\right)$ $-V_{i}$ separates $h$ from $k$ in $S-V_{i}$. There exists a continuum $N$ in $\left(F \cup I_{j}\right)-V_{i}$ which separates $h$ from $k$ in $S-V_{i}$ [6, Theorem 27, p. 177]. Let $B_{1}$ and $B_{2}$ be mutually exclusive arc-segments in $\mathrm{Bd} V_{2}$ which have endpoints $h$ and $k$. For $n=1$ and 2, there exists a point $e_{n}$ in $B_{n} \cap N$. The points $e_{1}$ and $e_{2}$ are contained in distinct components of $N-I_{j}[6$, Theorem 28, p. 156]. It follows that for $n=1$ and 2, $\left\{a_{j}, b_{j}\right\}$ meets the $e_{n}$-component of $N-I_{j}$ at exactly one point. The $\theta$-curve $L \cup B d V_{i}$ separates $a_{j}$ from $b_{j}$ in $S$ [6, Theorem 28, p. 156]. But since $H$ is a continuum in $S-\left(L \cup \operatorname{Bd} V_{i}\right)$ containing $\left\{a_{j}, b_{j}\right\}$, this is a contradiction. Hence $\left\{c_{1}, c_{2}, c_{3}, \cdots\right\} \cap D$ is finite.

It follows from the same argument that $\left\{d_{1}, d_{2}, d_{3}, \cdots\right\} \cap D$ is finite (the roles of the sequences $c_{1}, c_{2}, c_{3}, \cdots$ and $d_{1}, d_{2}, d_{3}, \cdots$ are interchanged). Hence there exist integers $i$ and $j(i<j)$ such that $\left\{c_{i}, d_{i}, c_{j}, d_{j}\right\}$ is contained in $S-M$. By the argument in the last part of the preceding paragraph, $F \cup I_{j}$ does not separate either $c_{i}$ from $c_{j}$ or $d_{i}$ from $d_{j}$ in $S$. There exist arcs $X$ and $Y$ in $S-\left(F \cup I_{j}\right)$ such that $X$ has endpoints $c_{i}$ and $c_{j}$ and $Y$ has endpoints $d_{i}$ and $d_{j}$. It follows that $X \cup Y \cup T_{i} \cup T_{j}$ contains a simple closed curve $J$ which 
separates $a_{j}$ from $b_{j}$ [6, Theorem 32, p. 181]. Since $J$ does not meet $F$, this is a contradiction. Hence $M$ is not aposyndetic at $x$ with respect to $y$.

Definition. A point $y$ of a continuum $M$ cuts $x$ from $z$ in $M$ (cuts $M$ between $x$ and $z$ ) if $x, y$, and $z$ are distinct points of $M$ and $y$ belongs to each subcontinuum of $M$ which contains $\{x, z\}$.

THEOREM 2. If $M$ is a bounded semi-aposyndetic plane continuum which does not have infinitely many complementary domains, then $M$ is arcwise connected.

Proof. Let $P$ be the set consisting of all natural numbers $n$ such that if $M$ is a semi-aposyndetic continuum in $S$ and $S-M$ has exactly $n$ components, then $M$ is arcwise connected. The natural number 1 belongs to $P[3]$. Assume $1,2, \cdots, m$ belong to $P$. Let $M$ be a semi-aposyndetic continuum in $S$ such that $S-M$ has exactly $m+1$ components. Let $p$ and $q$ be distinct points of $M$. Let $G$ denote a component of $S-M$ and define $C$ to be $S-(M \cup G)$. Suppose $\mathrm{Cl} G \cap \mathrm{Cl} C$ is totally disconnected. There exists a simple closed curve $J$ in $M$ which separates $G$ from a component of $C$ [7, Theorem 3.1, p. $108]$. Let $K$ and $L$ be the complementary domains of $J$. It follows from Theorem 1 that $(\mathrm{Cl} K \cap M) \cup L$ and $(\mathrm{Cl} L \cap M) \cup K$ are semiaposyndetic continua in $S$ each having no more than $m$ complementary domains. By the induction assumption, there exist arcs $I$ and $B$ in $(\mathrm{Cl} K \cap M) \cup L$ and $(\mathrm{Cl} L \cap M) \cup K$ which go from $p$ to $q$. In $(J \cup I \cup B) \cap M$ there exists an arc with endpoints $p$ and $q$.

Suppose $\mathrm{Cl} G \cap \mathrm{Cl} C$ has a nondegenerate component $Q$. If $Q$ $-\{p, q\}$ contains a point $x$ which does not cut $M$ between $p$ and $q$, then in $S$ there is a circular region $D$ containing $x$ such that $\{p, q\}$ is a subset of a component $F$ of $M-D . F$ is a semi-aposyndetic continuum (Theorem 1 ) in $S$ which has no more than $m$ complementary domains. Hence $F$ contains an arc with endpoints $p$ and $q$. Suppose each point of $Q-\{p, q\}$ cuts $M$ between $p$ and $q$. Let $Z$ be the set of all points which cut $p$ from $q$ in $M$. There exists an $\operatorname{arc} A$ (not necessarily in $S$ ) from $p$ to $q$ containing $Z$ such that if $x$ is a point of $Z$, then $x$ does not cut $M$ either between two points of ( $p$ component of $A-\{x\}) \cap(Z \cup\{p\})$ or between two points of $(q$ component of $A-\{x\}) \cap(Z \cup\{q\})$ [3 (in the theorem's proof)]. Let $T$ be a subarc of $(A-\{p, q\}) \cap Q$. Let $r$ and $t$ denote the endpoints of $T$ and assume without loss of generality that $t$ follows $r$ with respect to the order on $A$. Let $x$ be a point of $T-\{r, t\}$. There exists a circular region $U$ in $S$ containing $x$ such that $H$ (the $r$-component of $M-U$ ) contains $p$ and $Y$ (the $t$-component of $M-U$ ) contains $q$. $H$ and $Y$ 
are semi-aposyndetic continua (Theorem 1) in $S$ and the sets $S-H$ and $S-Y$ each have no more than $m$ components. It follows that $H \cup Y \cup T$ contains an arc which has endpoints $p$ and $q$. Evidently $M$ is arcwise connected. Hence $m+1$ belongs to $P$. By the Second Principle of Induction, all natural numbers belong to $P$.

REMARK. Let $M$ be a bounded plane continuum which does not separate the plane. In [5], it is proved that $M$ has Jones's cyclic property (that is, if $p$ and $q$ are distinct points of $M$ and no point cuts $p$ from $q$ in $M$, then there exists a simple closed curve lying in $M$ which contains $p$ and $q$ ). For a related theorem see [1, Theorem 2]. Also in [5], there is an example of a bounded plane continuum which has two complementary domains and fails to have this cyclic property. However, this continuum is not semi-aposyndetic. Using Theorem 1 and Theorem 2, one can easily establish Jones's cyclic property for semi-aposyndetic bounded plane continua which do not have infinitely many complementary domains.

\section{BIBLIOGRAPHY}

1. C. L. Hagopian, Concerning the cyclic connectivity of plane continua, Michigan Math. J. (to appear).

2. - Semiaposyndetic nonseparating plane continua are arcwise connected, Bull. Amer. Math. Soc. 77 (1971), 593-595.

3. - An arc theorem for plane continua, Illinois J. Math. (to appear).

4. F. B. Jones, Aposyndetic continua and certain boundary problems, Amer. J. Math. 63 (1941), 545-553. MR 3, 59.

5. - The cyclic connectivity of plane continua, Pacific J. Math. 11 (1961), 1013-1016. MR 25 \#2583.

6. R. L. Moore, Foundations of point set theory, rev. ed., Amer. Math. Soc. Colloq. Publ., vol. 13, Amer. Math. Soc., Providence, R. I., 1962.

7. G. T. Whyburn, Analytic topology, Amer. Math. Soc. Colloq. Publ., vol. 28, Amer. Math. Soc., Providence, R. I., 1963. MR 32 \#425.

Sacramento State College, Sacramento, California 95819 